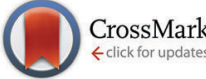

Cite this: Phys. Chem. Chem. Phys., 2014, 16, 18141

\section{Hydrogen bonding and proton transfer in cocrystals of 4,4'-bipyridyl and organic acids studied using nuclear quadrupole resonance}

\begin{abstract}
Janez Seliger*ab and Veselko Žagar ${ }^{a}$
Cocrystals of 4,4'-bipyridyl and several carboxylic acids were grown from the methanol solution of the cocrystal formers. Complete ${ }^{14} \mathrm{~N}$ NQR spectra of these cocrystals have been measured using ${ }^{1} \mathrm{H}-{ }^{14} \mathrm{~N}$ nuclear quadrupole double resonance. The principal values of the quadrupole coupling tensor are calculated from the ${ }^{14} \mathrm{~N} N Q R$ frequencies. A large variation in the ${ }^{14} \mathrm{~N}$ quadrupole coupling constant between $1.3 \mathrm{MHz}$ and $4.7 \mathrm{MHz}$ is observed. A very low ${ }^{14} \mathrm{~N}$ quadrupole coupling constant, characteristic for proton transfer $\mathrm{O}-\mathrm{H} \cdots \mathrm{N} \rightarrow \mathrm{O}^{-} \cdots \mathrm{H}-\mathrm{N}^{+}$, is observed in 4,4'-bipyridyl-oxalic acid (1:1). In 4,4'-bipyridyl-5chlorosalycilic acid $(1: 1)$ the ${ }^{14} \mathrm{~N} N Q R$ data show the presence of a short, strong $\mathrm{N} \ldots \mathrm{H} \ldots \mathrm{O}$ hydrogen bond. A correlation of the principal values of the ${ }^{14} \mathrm{~N}$ quadrupole coupling tensor is observed. The correlation is analyzed in the model, where the deformation of the lone pair electron orbital and the change of the population of the $\pi$-electron orbital produce the variation of the ${ }^{14} \mathrm{~N}$ quadrupole coupling tensor in the hydrogen bonded 4,4'-bipyridyl. The temperature variation of the ${ }^{14} \mathrm{~N}$ quadrupole coupling tensor in 4,4'-bipyridyl-5-chlorosalycilic acid $(1: 1)$ is analyzed. Proton displacement within the N . . H ..O hydrogen bond and the change of the population of the $\pi$-electron orbital at the two nitrogen positions in a 4,4'-bipyridyl molecule in the temperature interval between $157 \mathrm{~K}$ and $323 \mathrm{~K}$ are determined.
\end{abstract}

Received 17th June 2014, Accepted 8th July 2014

DOI: $10.1039 / \mathrm{c} 4 \mathrm{cp} 02665 a$

www.rsc.org/pccp

\section{Introduction}

Crystal engineering is the understanding of intermolecular interactions in the context of crystal packing and the utilization of such understanding in the design of new solids with desired physical, chemical and pharmaceutical properties. ${ }^{1}$ It is an interplay between the structure and properties of molecules on one hand and those of extended assemblies of molecules on the other. More precisely, one attempts to understand crystal structure and properties in terms of molecular structure and intermolecular interactions.

Cocrystals are often used in crystal engineering. A cocrystal is a nonionic supramolecular complex, which is constructed through several types of interaction, including hydrogen bonding, $\pi-\pi$ stacking, and van der Waals forces. ${ }^{2-4}$ In a cocrystal, homosynthons and heterosynthons generally occur. Their occurrence depends on the molecular architecture and the positions and properties of the functional groups.

Several cocrystals and crystals may exist in more than one crystal structure. These polymorphic forms of a given substance

\footnotetext{
a "Jozef Stefan" Institute, Jamova 39, 1000 Ljubljana, Slovenia

${ }^{b}$ Faculty of Mathematics and Physics, University of Ljubljana, Jadranska 19,

1000 Ljubljana, Slovenia. E-mail: janez.seliger@fmf.uni-lj.si;

Fax: +3861 2517281; Tel: +38614766576
}

in general differ in the macroscopic properties. Various polymorphs can be obtained by changing the conditions (solvent, temperature, pressure...) during the crystallization.

4,4'-Bipyridyl (BPY, Fig. 1) is a double hydrogen bond acceptor. It is thus a suitable compound to form hydrogen<smiles>c1cc(-c2ccncc2)ccn1</smiles>

4,4'-bipyridyl(BPY)<smiles>O=C(O)c1ccc(O)cc1</smiles>

4-hydroxybenzoic acid (4HBA)<smiles>O=C(O)C(=O)O</smiles>

oxalic acid (OXA)<smiles>O=C(O)c1ccccc1</smiles>

benzoic acid (BA)<smiles>O=C(O)c1cc(Cl)ccc1O</smiles>

Fig. 1 Structural formulae of cocrystal formers. 
bonded cocrystals with organic acids. Several cocrystals of 4,4'-bipyridyl have already been studied.

The crystal structure of the hydrogen bonded, 1:1 molecular complex of malonic acid with 4,4'-bipyridyl has been determined. Molecular tape structures are formed where the molecules are bound through strong $\mathrm{O}-\mathrm{H} \cdots \mathrm{N}$ and weak $\mathrm{C}-\mathrm{H} \cdots \mathrm{O}$ hydrogen bonds. The molecular tapes are arranged in a crossed fashion. ${ }^{5}$

BPY and oxalic acid crystallize in a 1:1 ratio. The asymmetric unit consists of one oxalic acid molecule and one BPY molecule in general positions, together with one half oxalic acid molecule and one half BPY molecule; the latter two molecules are centrosymmetric. The molecules are linked in two parallel independent chains by strong $\mathrm{O}-\mathrm{H} \cdots \mathrm{N}$ hydrogen bonds. In one chain there is one independent $\mathrm{O}-\mathrm{H} \cdots \mathrm{N}$ hydrogen bond and the molecules lie on the centres of symmetry. The second chain contains two independent $\mathrm{O}-\mathrm{H} \cdots \mathrm{N}$ hydrogen bonds and both molecules are twisted about their central bonds. ${ }^{6}$

The $1: 2$ adduct of benzene-1,2,4,5-tetracarboxylic acid and BPY has been studied using single-crystal neutron diffraction. The structure contains at $20 \mathrm{~K}$ two short, strong $\mathrm{N} \cdots \mathrm{O}$ hydrogen bonds: one $\mathrm{O}-\mathrm{H} \cdots \mathrm{N}$ hydrogen bond and one $\mathrm{N}-\mathrm{H} \cdots \mathrm{O}$ hydrogen bond. The $\mathrm{N}-\mathrm{H}$ distance in the strong, short $\mathrm{N}-\mathrm{H} \cdots \mathrm{O}$ hydrogen bond changes from $1.207 \AA$ at $20 \mathrm{~K}$ to $1.302 \AA 296 \mathrm{~K}$ $(\mathrm{N}-\mathrm{H} \cdots \mathrm{O} \rightarrow \mathrm{N} \cdots \mathrm{H}-\mathrm{O})$. At $200 \mathrm{~K}$ the $\mathrm{H}$ atom lies in an intermediate position between $\mathrm{N}$ and $\mathrm{O}$. The $\mathrm{O}-\mathrm{H} \cdots \mathrm{N}$ hydrogen bond, on the other hand, does not change with temperature. ${ }^{7}$

2,5-Dihydroxy-1,4-benzoquinone and BPY crystallize in a $1: 1$ ratio as a neutral molecular adduct in the space group $C 2 / c$, with half of each molecule in the asymmetric unit. The molecules are linked by strong $\mathrm{O}-\mathrm{H} \cdots \mathrm{N}$ hydrogen bonds and weak $\mathrm{C}-\mathrm{H} \cdots \mathrm{O}$ hydrogen bonds to form infinite stacks of parallel onedimensional hydrogen bonded ribbons. ${ }^{8}$

BPY and 2,5-dichloro-3,6-dihydroxy-1,4-benzoquinone (chloranilic acid) crystallize in a 1:1 ratio. The two molecules form bifurcated hydrogen bonds and link as molecular chains. ${ }^{9}$

In the $1: 1$ adduct of $4,4^{\prime}$-dihydroxybenzophenone and BPY the components are linked into chains by $\mathrm{O}-\mathrm{H} \cdots \mathrm{N}$ hydrogen bonds. In the 2:3 adduct formed between 1,3,5-trihydroxybenzene and BPY, pairs of molecules of each component are linked by $\mathrm{O}-\mathrm{H} \cdots \mathrm{N}$ hydrogen bonds into macrocyclic rings, which are themselves linked by further BPY molecules into chains of rings; these chains are cross-linked by $\mathrm{C}-\mathrm{H} \cdots \mathrm{O}$ hydrogen bonds. ${ }^{10}$

BPY and 4-hydroxybenzoic acid (4HBA) crystallize in $1: 2$ and $2: 1$ ratios. Cocrystals of BPY and 4-hydroxybenzoic acid $(1: 2)$ show synthon polymorphism. In Form 1 the phenolic $\mathrm{OH}$ groups of two repetitive 4HBA molecules in a chain form $\mathrm{O}-\mathrm{H} \cdots \mathrm{N}$ hydrogen bonds to two BPY molecules, whereas the carboxyl groups of the two 4HBA molecules form a pair of $\mathrm{O}-\mathrm{H} \cdots \mathrm{O}$ hydrogen bonds. In Form 2 the two 4HBA molecules between two BPY molecules are oriented in the same sense so that the phenolic $\mathrm{OH}$ group of the first molecule and the carboxyl $\mathrm{OH}$ group of the second molecule form $\mathrm{O}-\mathrm{H} \cdots \mathrm{N}$ hydrogen bonds to the two BPY molecules. The phenolic $\mathrm{OH}$ group of the second 4HBA molecule forms an $\mathrm{O}-\mathrm{H} \cdots \mathrm{O}$ hydrogen bond to the carbonyl oxygen of the first $4 \mathrm{HBA}$ molecule.
In the $2: 1$ cocrystal a $4 \mathrm{HBA}$ molecule is sandwiched between two BPY molecules and forms two $\mathrm{O}-\mathrm{H} \cdots \mathrm{N}$ hydrogen bonds. The structural roles of the two hydrogen bonded bipyridine $\mathrm{N}$-atoms are distinct. ${ }^{11}$

Nuclear quadrupole resonance (NQR) has proven to be a sensitive technique for the study of molecular electron configuration in solid materials. In hydrogen bonded solids the change of the electron configuration produced by hydrogen bonding causes a shift of the NQR frequencies. ${ }^{12}$ It has recently been shown how NQR can be used to investigate cocrystals and crystal polymorphs. ${ }^{13,14}$

Two studies of cocrystals of 2-amino-4,6-dimethylpyrimidine and 2,3,5,6-tetramethylpyrazine on one side and several carboxylic acids on the other side have already been performed. ${ }^{15,16}$

In the present paper we present the results of NQR investigation of cocrystals of BPY and benzoic acid (BA), 5-chlorosalycilic acid (5CSA), 4-hydroxybenzoic acid (4HBA), oxalic acid (OXA) and malonic acid (MA). The structural formulae of the molecules used in the present study are presented in Fig. 1.

In the compounds studied there are two quadrupole atomic nuclei, ${ }^{14} \mathrm{~N}$ and ${ }^{17} \mathrm{O}$, which are suitable for the investigation of hydrogen bonds. Due to experimental reasons we decided to use ${ }^{14} \mathrm{~N} N Q R$ to characterize the cocrystals and to investigate the influence of hydrogen bonding on the electron charge distribution at the positions of the nitrogen atoms and possible proton transfer in these systems.

\section{${ }^{14} \mathrm{~N} N Q R$}

The nucleus of the nitrogen isotope ${ }^{14} \mathrm{~N}$ has in its ground state a spin of $I=1$ and a nonzero electric quadrupole moment. The interaction of the nuclear electric quadrupole moment $e Q$ with the electric field gradient (EFG) tensor $V_{\mathrm{ik}}, V_{\mathrm{ik}}=\partial^{2} V / \partial x_{\mathrm{i}} \partial x_{\mathrm{k}}$, at the position of the atomic nucleus, results in three generally nonequidistant nuclear quadrupole energy levels. The three resonance (NQR) frequencies $\nu_{+} \geq \nu_{-} \geq \nu_{0}$ are expressed as ${ }^{17}$

$$
\nu_{ \pm}=\frac{e^{2} q Q}{4 h}(3 \pm \eta) \quad \nu_{0}=\nu_{+}-\nu_{-}=\frac{e^{2} q Q}{2 h} \eta
$$

Here, $e^{2} q Q / h$ is the quadrupole coupling constant (often abbreviated as qcc) and $\eta$ is the asymmetry parameter of the EFG tensor. They are related to the principal values $V_{X X}, V_{Y Y}$, and $V_{Z Z}$ of the EFG tensor $\left(\left|V_{Z Z}\right| \geq\left|V_{Y Y}\right| \geq\left|V_{X X}\right|\right)$ as $e^{2} q Q / h=\left|e Q V_{Z Z}\right| / h$ and $\eta=\left(V_{X X}-V_{Y Y}\right) / V_{Z Z}$. The quadrupole coupling tensor $q_{\mathrm{ik}}$, which can be determined by NQR or NMR, is equal to the product of the EFG tensor and the nuclear quadrupole moment $e Q$ divided by the Planck constant $h, q_{\mathrm{ik}}=e Q V_{\mathrm{ik}} / h$. It is expressed in frequency units. The present knowledge of nuclear electric quadrupole moments ${ }^{18}$ allows us to calculate the EFG tensor from the quadrupole coupling tensor. The sign of the largest principal value $q_{z z}$ of the quadrupole coupling tensor can in general not be determined by NQR or NMR, so only the relative signs of the elements of the quadrupole coupling tensor with respect to $q_{z z}$ can be determined by these techniques. Different experimental techniques, as for example microwave spectroscopy, or quantum chemical calculations are needed to 
determine its sign. The absolute value of the largest principal value of the ${ }^{14} \mathrm{~N}$ quadrupole coupling tensor and the asymmetry parameter $\eta$ are calculated from the ${ }^{14} \mathrm{~N} N Q R$ frequencies as $\left|q_{z Z}\right|=e^{2} q Q / h=2\left(\nu_{+}+\nu_{-}\right) / 3$ and $\eta=2 \nu_{0} /\left|q_{z z}\right|$. The two smaller principal values of the quadrupole coupling tensor are related to $q_{Z Z}$ and $\eta$ as $q_{Y Y}=-q_{Z Z}(1+\eta) / 2$ and $q_{X X}=-q_{Z Z}(1-\eta) / 2$.

\section{Experimental section}

The samples of BPY, benzoic acid, 5-chlorosalycilic acid, 4-hydroxybenzoic acid, oxalic acid and malonic acid were purchased at Sigma-Aldrich and used as obtained. The cocrystals were obtained by mixing hot methanol solutions $\left(\sim 50{ }^{\circ} \mathrm{C}\right)$ of cocrystal formers. The solutions were then left at room temperature for a few days until the cocrystals grew. In the case of BPY-OXA $(1: 1)$ the solid immediately precipitated from the solution. The obtained solid is not soluble in methanol. We dissolved a part of the obtained solid in hot water, cooled the solution to room temperature and left it at this temperature for several days until the cocrystals grew.

The ${ }^{14} \mathrm{~N}$ NQR frequencies have been measured by ${ }^{1} \mathrm{H}^{-14} \mathrm{~N}$ nuclear quadrupole double resonance (NQDR). In the present study, we have used the solid-effect technique ${ }^{19}$ and the technique using multiple frequency sweeps and two-frequency irradiation..$^{20,21}$ These techniques are based on magnetic field cycling. The details of the present experimental setup and the measuring procedure were published in a previous paper. ${ }^{22}$

\section{Results and discussion}

The ${ }^{1} \mathrm{H}^{-14} \mathrm{~N}$ NQDR spectrum of BPY-BA $(1: 1)$ is presented in Fig. 2.

We observe two sets of ${ }^{14} \mathrm{~N}$ NQR frequencies $\left(\nu_{+}, \nu_{-}, \nu_{0}\right)$ : $(4020 \mathrm{kHz}, 3070 \mathrm{kHz}, 950 \mathrm{kHz})$ and $(3250 \mathrm{kHz}, 2630 \mathrm{kHz}, 620 \mathrm{kHz})$. The frequencies in the first set are close to the ${ }^{14} \mathrm{~N}$ NQR frequencies observed in solid pyridine ${ }^{23}$ and may be assigned to the nonhydrogen bonded nitrogen position. The ${ }^{14} \mathrm{~N}$ NQR frequencies in the second set are significantly lower and may be assigned to the nitrogen atom which is hydrogen bonded to carboxylic $\mathrm{OH}$ of benzoic acid.

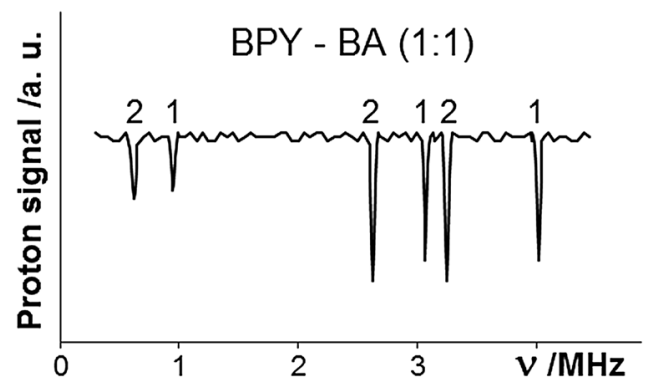

Fig. $2{ }^{1} \mathrm{H}-{ }^{14} \mathrm{~N}$ NQDR spectrum of $4,4^{\prime}$-bipyridyl-benzoic acid $(1: 1)$ at $T=295 \mathrm{~K}$. The lines labeled by " 1 " correspond to the non-hydrogenbonded nitrogen position whereas the lines labeled by " 2 " correspond to the hydrogen-bonded nitrogen position.

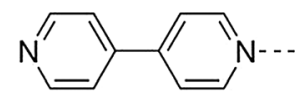

(a)

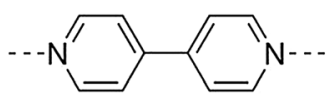

(b)

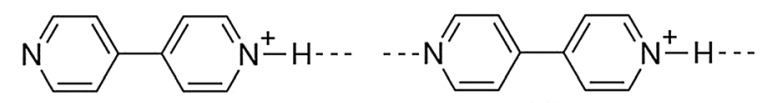

(c)

(d)

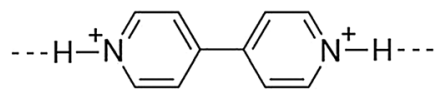

(e)

Fig. 3 Possible hydrogen bonding schemes of BPY.

The possible hydrogen bonding schemes of a BPY molecule are presented in Fig. 3.

Scheme (a) is present in BPY-BA $(1: 1)$. Within the experimental resolution of NQDR it may be also concluded that all BPY molecules are crystallographically equivalent.

A similar situation is observed in BPY-5CSA (1:1), where we at room temperature observe two sets of ${ }^{14} \mathrm{~N}$ NQR frequencies: $(3882 \mathrm{kHz}, 2982 \mathrm{kHz}, 900 \mathrm{kHz})$ and $(2010 \mathrm{kHz}, 1866 \mathrm{kHz}$, $144 \mathrm{kHz}$ ). The ${ }^{14} \mathrm{~N}$ NQR frequencies in the first set are characteristic for a non-hydrogen bonded nitrogen position. The ${ }^{14} \mathrm{~N} N Q R$ frequencies in the second set are rather low. They are in the range of ${ }^{14} \mathrm{~N} N Q R$ frequencies observed in pyridine-3,5dicarboxylic acid, where short, strong $\mathrm{N} \cdots \mathrm{H} \cdots \mathrm{O}$ hydrogen bonds are formed. ${ }^{24}$ In this system, by varying temperature, phonon driven proton transfer occurs. ${ }^{25,26}$ At low temperature proton is closer to the hydrogen bond acceptor than to the hydrogen bond donor $\left(\mathrm{N}^{+}-\mathrm{H} \cdots \mathrm{O}^{-}\right)$. Upon increasing temperature proton is continuously displaced from the hydrogen bond acceptor to the hydrogen bond donor $\left(\mathrm{N}^{+}-\mathrm{H} \cdots \mathrm{O}^{-} \rightarrow \mathrm{N} \cdots \mathrm{H}-\mathrm{O}\right)$. The proton displacement results in a strong temperature variation of the ${ }^{14} \mathrm{~N}$ NQR frequencies. Due to the possibility that a similar effect also occurs in BPY-5CSA (1:1) we

Table $1{ }^{14} \mathrm{~N}$ NQR frequencies, quadrupole coupling constants $e^{2} q Q / h$ and asymmetry parameters $\eta$ in cocrystals of BPY and carboxylic acids

\begin{tabular}{|c|c|c|c|c|c|c|c|}
\hline Substance & $\begin{array}{l}T \\
(\mathrm{~K})\end{array}$ & $\begin{array}{l}\text { Nitrogen } \\
\text { position }\end{array}$ & $\begin{array}{l}\nu_{+} \\
(\mathrm{kHz})\end{array}$ & $\begin{array}{l}\nu_{-} \\
(\mathrm{kHz})\end{array}$ & $\begin{array}{l}\nu_{0} \\
(\mathrm{kHz})\end{array}$ & $\begin{array}{l}e^{2} q Q / h \\
(\mathrm{kHz})\end{array}$ & $\eta$ \\
\hline \multirow[t]{6}{*}{ BPY-BA $(1: 1)$} & \multirow[t]{2}{*}{295} & $\mathrm{~N} \cdots \mathrm{H}-\mathrm{O}$ & 3250 & 2630 & 620 & 3920 & 0.316 \\
\hline & & $\mathrm{N}$ & 4020 & 3070 & 950 & 4727 & 0.402 \\
\hline & \multirow[t]{2}{*}{323} & $\mathrm{~N} \cdots \mathrm{H} \cdots \mathrm{O}$ & 2040 & 1885 & 155 & 2617 & 0.118 \\
\hline & & $\mathrm{N}$ & 3867 & 2979 & 888 & 4564 & 0.389 \\
\hline & \multirow[t]{2}{*}{295} & $\mathrm{~N} \cdots \mathrm{H} \cdots \mathrm{O}$ & 2010 & 1866 & 144 & 2584 & 0.111 \\
\hline & & $\mathrm{N}$ & 3882 & 2982 & 900 & 4576 & 0.393 \\
\hline \multirow[t]{6}{*}{ BPY-5CSA (1:1) } & \multirow[t]{2}{*}{228} & $\mathrm{~N} \cdots \mathrm{H} \cdots \mathrm{O}$ & 1981 & 1843 & 138 & 2549 & 0.108 \\
\hline & & $\mathrm{N}$ & 3910 & 2989 & 921 & 4599 & 0.400 \\
\hline & \multirow[t]{2}{*}{193} & $\mathrm{~N} \cdots \mathrm{H} \cdots \mathrm{O}$ & 1967 & 1842 & 126 & 2539 & 0.099 \\
\hline & & $\mathrm{N}$ & 3921 & 2995 & 926 & 4611 & 0.402 \\
\hline & \multirow[t]{2}{*}{157} & $\mathrm{~N} \cdots \mathrm{H} \cdots \mathrm{O}$ & 1957 & 1833 & 124 & 2527 & 0.098 \\
\hline & & $\mathrm{N}$ & 3928 & 2997 & 931 & 4617 & 0.403 \\
\hline BPY-OXA $(1: 1)$ & 179 & $\mathrm{~N}-\mathrm{H}^{+} \ldots \mathrm{O}$ & 1050 & 960 & 90 & 1340 & 0.134 \\
\hline \multirow[t]{2}{*}{ BPY-MA $(1: 1)$} & \multirow[t]{2}{*}{295} & $\mathrm{~N} \cdots \mathrm{H}-\mathrm{O}$ & 2930 & 2435 & 495 & 3577 & 0.277 \\
\hline & & $\mathrm{N} \cdots \mathrm{H}-\mathrm{O}$ & 3275 & 2670 & 605 & 3961 & 0.305 \\
\hline \multirow[t]{2}{*}{ BPY-4HBA $(2: 1)$} & \multirow[t]{2}{*}{181} & $\mathrm{~N} \cdots \mathrm{H}-\mathrm{O}$ & 3428 & 2733 & 695 & 4107 & 0.338 \\
\hline & & $\mathrm{N}$ & 3950 & 3000 & 950 & 4633 & 0.410 \\
\hline \multirow[t]{2}{*}{ BPY-4HBA $(1: 2)$} & \multirow[t]{2}{*}{175} & $\mathrm{~N} \cdots \mathrm{H}-\mathrm{O}$ & 3393 & 2715 & 678 & 4072 & 0.333 \\
\hline & & $\mathrm{N} \cdots \mathrm{H}-\mathrm{O}$ & 3410 & 2728 & 682 & 4092 & 0.333 \\
\hline
\end{tabular}


measured in this compound temperature dependences of the ${ }^{14} \mathrm{~N}$ NQR frequencies. The results of these measurements together with the other presently determined ${ }^{14} \mathrm{~N}$ NQR frequencies are presented in Table 1.

In BPY-4HBA $(2: 1)$ we performed the measurements at $T=181 \mathrm{~K}$ due to experimental reasons. Proton spin-lattice relaxation time $T_{1}$ at the Larmor frequency lower than $1 \mathrm{MHz}$ is too short at room temperature for the present experimental setup to be used. We therefore cooled the sample to a lower temperature to obtain a longer $T_{1}$. We observed three sets of ${ }^{14} \mathrm{~N}$ NQR frequencies: (3950 kHz, $3000 \mathrm{kHz}, 950 \mathrm{kHz}),(3428 \mathrm{kHz}$, $2733 \mathrm{kHz}, 695 \mathrm{kHz})$, and (3275 kHz, $2670 \mathrm{kHz}, 605 \mathrm{kHz})$. According to the crystal structure ${ }^{12}$ we expect to observe four sets of the ${ }^{14} \mathrm{~N}$ NQR frequencies: two from the hydrogen bonded nitrogen positions and two from the non-hydrogen bonded nitrogen positions (two nonequivalent BPY molecules with the hydrogen bond Scheme (a)). We observed only one nonhydrogen bonded position (the first set of NQR frequencies) so we suppose that the splitting of the ${ }^{14} \mathrm{~N}$ NQR frequencies from the two non-hydrogen bonded nitrogen positions is smaller than the experimental resolution of NQDR $(\sim 10 \mathrm{kHz})$. In our previous studies we also observed that a lower $\mathrm{p} K_{\mathrm{a}}$ of the hydrogen bond donor produces lower ${ }^{14} \mathrm{~N}$ NQR frequencies. We therefore assign the second set of ${ }^{14} \mathrm{~N}$ NQR frequencies to the nitrogen atom which is hydrogen bonded to the phenolic $\mathrm{OH}$ group and the third set of ${ }^{14} \mathrm{~N}$ NQR frequencies to the nitrogen atom which is hydrogen bonded to the carboxylic $\mathrm{OH}$ group.

In BPY-4HBA (1:2) we observe two slightly different sets of ${ }^{14} \mathrm{~N}$ NQR frequencies: (3410 kHz, $2728 \mathrm{kHz}, 682 \mathrm{kHz}$ ) and (3393 kHz, $2715 \mathrm{kHz}, 678 \mathrm{kHz}$ ). They are both approximately equal to the ${ }^{14} \mathrm{~N}$ NQR frequencies in BPY-4HBA $(2: 1)$ at the nitrogen position which is hydrogen bonded to the phenolic $\mathrm{OH}$ group. This situation is expected in polymorphic Form 1 with the hydrogen bonding Scheme (b). The polymorphic Form 2, where we expect two strongly different sets of ${ }^{14} \mathrm{~N}$ NQR frequencies, has not been observed within experimental accuracy. Most probably the mass fraction of this form in the polycrystalline sample is low, lower than approximately $20 \%$.

Two slightly different sets of ${ }^{14} \mathrm{~N}$ NQR frequencies in this compound show that there are two non-equivalent nitrogen positions in the unit cell. This is possible either if the two nitrogen positions in a BPY molecule are distinct or if there are two distinct centrosymmetric BPY molecules in the unit cell.

In BPY-OXA (1:1) we performed the NQDR measurements using the solid effect technique in the freshly prepared sample obtained by mixing hot methanol solutions of the cocrystal formers and in the sample obtained by recrystallization of this sample from the water solution. There is no difference in the NQDR spectra of the two samples. In both samples we observe a single set of rather low ${ }^{14} \mathrm{~N}$ NQR frequencies: $(1050 \mathrm{kHz}$, $960 \mathrm{kHz}, 90 \mathrm{kHz})$. Such low ${ }^{14} \mathrm{~N}$ NQR frequencies are characteristic for a pyridinium ion. ${ }^{27-31} \mathrm{NQR}$ data thus show that a double proton transfer from an oxalic acid molecule to two BPY molecules takes place and the hydrogen bonding scheme (e) occurs. Three distinct nitrogen positions, as observed by X-ray structure determination, ${ }^{7}$ have not been observed by NQDR.
Most probably the differences between the NQR frequencies are smaller than the experimental resolution of the solid effect technique $(\approx 20 \mathrm{kHz})$.

In the cocrystal BPY-MA $(1: 1)$ we observe only one set of ${ }^{14} \mathrm{~N}$ NQR frequencies at room temperature: $(2930 \mathrm{kHz}, 2435 \mathrm{kHz}$, $495 \mathrm{kHz}$ ). This means that a dicarboxylic malonic acid molecule forms two equivalent hydrogen bonds to two BPY molecules (Scheme (b)) in agreement with the crystal structure. ${ }^{5}$

The present NQDR measurements show a large variation of the ${ }^{14} \mathrm{~N}$ quadrupole coupling constant in hydrogen bonded BPY. It varies between $4.7 \mathrm{MHz}$ in the case of no hydrogen bond and $1.3 \mathrm{MHz}$ in the case of proton transfer. Hydrogen bonds between BPY and weaker carboxylic acids, BA $\left(\mathrm{p} K_{\mathrm{a}}=4.2\right)$ and 4HBA $\left(\mathrm{p} K_{\mathrm{a}}=4.5\right.$ (carboxylic $\mathrm{OH}$ ) and $\mathrm{p} K_{\mathrm{a}}=9.3$ (phenolic $\mathrm{OH})$ ), reduce the ${ }^{14} \mathrm{~N}$ quadrupole coupling constant to about $4 \mathrm{MHz}$. Hydrogen bonds between BPY and 5CSA $\left(\mathrm{p} K_{\mathrm{a}}=2.6\right)$ reduces the ${ }^{14} \mathrm{~N}$ quadrupole coupling constant to $2.6 \mathrm{MHz}$. In the case of dicarboxylic malonic acid $\left(\mathrm{p} K_{\mathrm{a}}=2.8\right.$ and 5.7) the two carboxylic $\mathrm{OH}$ groups form equivalent $\mathrm{O}-\mathrm{H} \cdots \mathrm{N}$ hydrogen bonds and the ${ }^{14} \mathrm{~N}$ quadrupole coupling constant reduces to 3.6 MHz. The strongest acid used in the present study, OXA $\left(\mathrm{p} K_{\mathrm{a}}=1.25\right.$ and 4.2$)$, produces a double proton transfer $\left(\mathrm{O}-\mathrm{H} \cdots \mathrm{N} \rightarrow \mathrm{O}^{-} \cdots \mathrm{H}-\mathrm{N}^{+}\right)$and reduction of $e^{2} q Q / h$ to $1.3 \mathrm{MHz}$.

It has been shown several times that the principal values of the ${ }^{14} \mathrm{~N}$ quadrupole coupling tensor in heterocyclic rings are correlated. ${ }^{12,15,16}$ A plot of $q_{X X}$ and $q_{Y Y}$ versus $q_{Z Z}$ in the presently studied compounds is presented in Fig. 4. The data for pyridine in the gas phase ${ }^{32}$ and the data for the pyridinium ion $^{27-31}$ are added. The diagram clearly shows that the correlation also exists in the present case. The correlations, presented as full lines on the diagram, are the following:

$$
\begin{aligned}
& q_{Y Y}=-0.70 \mathrm{MHz}-0.85 q_{Z Z} \\
& q_{X X}=+0.70 \mathrm{MHz}-0.15 q_{Z Z}
\end{aligned}
$$

The coefficients in the above expressions are not very much different from the coefficients obtained in the case of pyridine ${ }^{12}$ and 5,5'-dimethyl-2,2'-bipyridine. ${ }^{31}$ As already concluded, ${ }^{15}$ the hydrogen bond deforms the nitrogen lone pair orbital, which simultaneously produces a change of the population of the

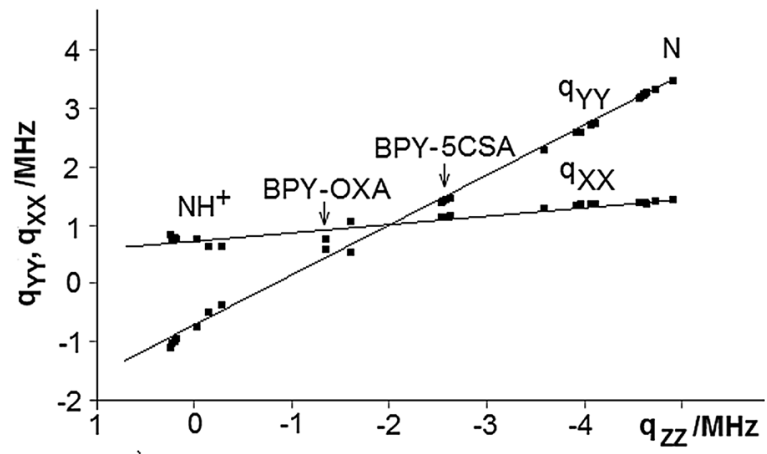

Fig. 4 Correlation of the principal values $q_{X X}, q_{Y Y}$, and $q_{Z Z}$ of the quadrupole coupling tensor in hydrogen bonded BPY. The data for pyridine in the gas phase (extremely right) and for the pyridinium ion (left, around $\left.q_{z z}=0 \mathrm{MHz}\right)$ are added. 
$\pi$ electron orbital. The two effects are correlated in the case of pyridine and pyrazine.

In substituted and hydrogen-bonded pyrimidine, we analyzed the ${ }^{14} \mathrm{~N}$ quadrupole coupling tensor in terms of the population of the $\pi$-electron orbital and deformation of the lone pair electron orbital. ${ }^{16}$ In the same model we can express the ${ }^{14} \mathrm{~N}$ quadrupole coupling tensor $\mathbf{q}$ in hydrogen bonded BPY as

$$
\begin{aligned}
\mathbf{q}= & \left(\begin{array}{ccc}
1.434 & 0 & 0 \\
0 & 3.474 & 0 \\
0 & 0 & -4.908
\end{array}\right) \mathrm{MHz}+x_{\mathrm{LP}}\left(\begin{array}{ccc}
-0.5 & 0 & 0 \\
0 & -0.5 & 0 \\
0 & 0 & 1
\end{array}\right) \\
& +x_{\pi}\left(\begin{array}{ccc}
-0.5 & 0 & 0 \\
0 & 1 & 0 \\
0 & 0 & -0.5
\end{array}\right)
\end{aligned}
$$

Here the first tensor contains the principal values of the ${ }^{14} \mathrm{~N}$ quadrupole coupling tensor of pyridine in the gas phase, $x_{\mathrm{LP}}$ is a parameter related to the deformation of the lone pair electron orbital and $x_{\pi}$ is proportional to the change of the population of the $\pi$-electron orbital with respect to an isolated pyridine molecule. Expressions (2) are obtained when $x_{\mathrm{LP}}=-1.64 x_{\pi}$. The principal values of the quadrupole coupling tensor are then expressed in terms of the parameter $x_{\pi}$ in the following way:

$$
\begin{gathered}
q_{X X}=1.434 \mathrm{MHz}+0.32 x_{\pi} \\
q_{Y Y}=3.474 \mathrm{MHz}+1.82 x_{\pi} \\
q_{Z Z}=-4.908 \mathrm{MHz}-2.14 x_{\pi}
\end{gathered}
$$

In the case of a full proton transfer (pyridinium ion) $q_{z z}$ is approximately zero. This corresponds to $x_{\pi}=-2.3 \mathrm{MHz}$. If we assume that the largest principal value of the axially symmetric contribution of one electron in the nitrogen $2 \mathrm{p}$ orbital to the quadrupole coupling tensor is $-9 \mathrm{MHz}^{27}$ we obtain from the NQR data an increase of the population of the nitrogen $\pi$-electron orbital equal to $(-2.3 \mathrm{MHz}) /(-9 \mathrm{MHz})=0.25$.

The data for the hydrogen bonded nitrogen position in BPY-5CSA are approximately in the center of the correlation diagram. This position indicates the existence of a short, strong $\mathrm{N} \cdots \mathrm{H} \cdots \mathrm{O}$ hydrogen bond. In pyridine-3,5-dicarboxylic acid, where a strong short $\mathrm{N} \cdots \mathrm{H} \cdots \mathrm{O}$ hydrogen bond is formed, proton continuously migrates from the $\mathrm{N}^{+}-\mathrm{H} \cdots \mathrm{O}^{-}$position at low temperature to the $\mathrm{N} \cdot \mathrm{H}-\mathrm{O}$ position at high temperature. $^{25,26}$ The NQR measurements ${ }^{24}$ show a continuous temperature variation of the ${ }^{14} \mathrm{~N}$ quadrupole coupling constant $e^{2} q Q / h$. The ratio $\Delta R(\mathrm{~N} \cdots \mathrm{H}) / \Delta e^{2} q Q / h$ is equal to $0.36 \AA \mathrm{MHz}^{-1}$. Here $\Delta R(\mathrm{~N} \cdots \mathrm{H})$ is the change of the $\mathrm{N} \cdots \mathrm{H}$ distance and $\Delta e^{2} q Q / h$ is the corresponding change of the ${ }^{14} \mathrm{~N}$ quadrupole coupling constant. The ${ }^{14} \mathrm{~N}$ NQR frequencies in BPY-5CSA are very close to the ${ }^{14} \mathrm{~N}$ NQR frequencies in pyridine-3,5-dicarboxylic acid so we assume that the ratio $\Delta R(\mathrm{~N} \cdots \mathrm{H}) / \Delta e^{2} q Q / h$ is the same in both compounds. Upon increasing the temperature from $157 \mathrm{~K}$ to $323 \mathrm{~K}$ the ${ }^{14} \mathrm{~N}$ quadrupole coupling constant increases for
$\Delta e^{2} q Q / h=90 \mathrm{kHz}$, so the nitrogen-proton distance $R(\mathrm{~N} \cdots \mathrm{H})$ increases for $0.03 \AA$ A. The actual increase of $R(\mathrm{~N} \cdots \mathrm{H})$ may be somewhat larger, because there are two competing effects producing the temperature variation of $e^{2} q Q / h$ at this nitrogen position. Proton displacement towards oxygen increases $e^{2} q Q / h$, whereas molecular thermal motion, which amplitude increases with increasing temperature, produces a decrease of $e^{2} q Q / h$ when temperature increases. If we suppose that the proton displacement dominates the temperature variation of $e^{2} q Q / h$ we may conclude that in BPY-5CSA the proton displacement is in the temperature interval between $157 \mathrm{~K}$ and $323 \mathrm{~K}$ about four times smaller than in pyridine-3,5-dicarboxylic acid.

To check whether in this compound the proton displacement at the hydrogen bonded nitrogen position influences the electron charge distribution at the non-hydrogen bonded nitrogen position in BPY and to get some more information on thermal motions, we plot the temperature variation of $q_{Y Y}$ versus $q_{z Z}$ for both nitrogen positions. The plot is presented in Fig. 5. The correlation line (expression 2) is also shown in the diagram.

At the hydrogen bonded nitrogen position $q_{Z Z}$ and $q_{Y Y}$ increase in magnitude with increasing temperature. The experimental points are located on a line, which is nearly parallel to the correlation line. The distance between the two lines is small. This demonstrates that the temperature variation of the hydrogen bond parameters dominates the temperature variation of the ${ }^{14} \mathrm{~N}$ quadrupole coupling tensor at this nitrogen position.

At the non-hydrogen bonded nitrogen position $q_{Z Z}$ and $q_{Y Y}$ decrease in magnitude with increasing temperature. The change of $e^{2} q Q / h$ at the non-hydrogen bonded nitrogen position is equal to $60 \%$ of the change of $e^{2} q Q / h$ at the hydrogen bonded nitrogen
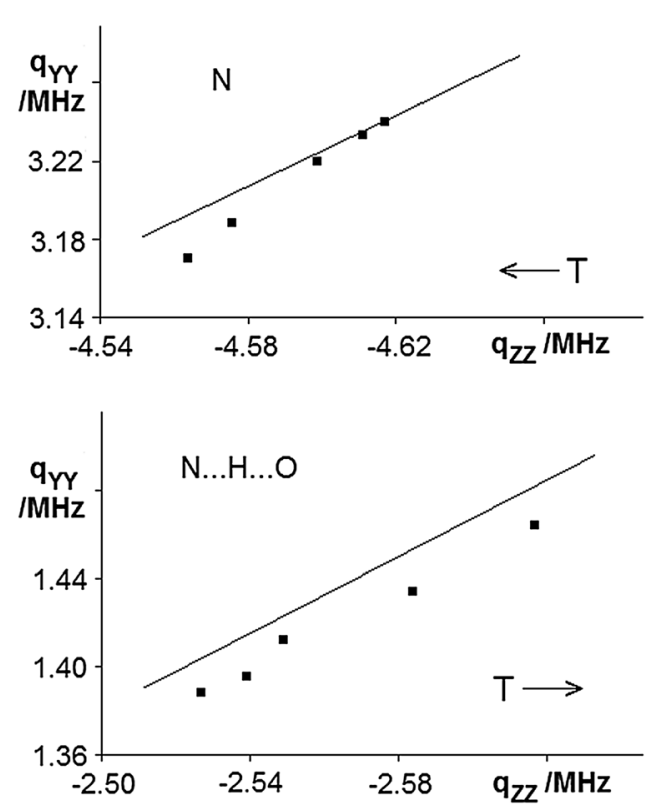

Fig. 5 Temperature variation of the principal values $q_{Z Z}$ and $q_{Y Y}$ of the quadrupole coupling tensor at the two nitrogen positions in BPY-5CSA $(1: 1)$. The solid lines are two parts of the correlation line ( $q_{Y y}$ versus $\left.q_{z z}\right)$ given by expression 2 . 
position in the same temperature interval. The line through the experimental points is not parallel to the correlation line. This may be the effect of thermal motions. We may assume that the dominant thermal motion of a BPY molecule is libration of pyridine rings around the long molecular axis. This motion does not change $q_{z Z}$. The principal axis $Z$ is parallel to the long molecular axis. The libration only reduces the difference between $q_{Y Y}$ and $q_{X X}$.

We define the molecule-fixed coordinate system $(X, Y, Z)$ along the principal directions of the ${ }^{14} \mathrm{~N}$ quadrupole coupling tensor for a static molecule. In addition we define the crystalfixed coordinate system $(x, y, z=Z)$ in such a way that the molecular axes $X$ and $Y$ are time average directed along the crystal-fixed axes $x$ and $y$ respectively. The libration around the $z$ axis produces time variation of $q_{X X}, q_{Y Y}$ and $q_{x y}$. The principal values of the time-averaged quadrupole coupling tensor $\langle\mathbf{q}\rangle$, that are observed by NQR, are in the case of small librations equal to

$$
\begin{gathered}
\langle q\rangle_{z z}=q_{z z} \\
\langle q\rangle_{y y}=q_{Y Y}-\left(q_{Y Y}-q_{X X}\right)\left\langle\phi^{2}\right\rangle \\
\langle q\rangle_{x x}=q_{X X}+\left(q_{Y Y}-q_{X X}\right)\left\langle\phi^{2}\right\rangle
\end{gathered}
$$

Here $\left\langle\phi^{2}\right\rangle$ is the mean square libration angle. For the nonhydrogen bonded nitrogen position the difference $q_{Y Y}-q_{X X}$ is approximately equal to $1.8 \mathrm{MHz}$, whereas for the hydrogen bonded nitrogen position it is equal to $0.3 \mathrm{MHz}$. The line through the experimental points at the non-hydrogen bonded nitrogen position becomes parallel to the correlation line when we add the librations in such a way that the following relation holds

$$
\left(q_{Y Y}-q_{X X}\right)\left(\left\langle\phi^{2}\right\rangle_{295 \mathrm{~K}}-\left\langle\phi^{2}\right\rangle_{157 \mathrm{~K}}\right)=20 \mathrm{kHz}
$$

The mean square of the libration angle $\left\langle\phi^{2}\right\rangle$ is usually proportional to the absolute temperature. If the same holds in the present compound we calculate from expression (6) $\left\langle\phi^{2}\right\rangle_{295 \mathrm{~K}}$ as being equal to $0.022 \mathrm{rad}^{2}$. Its square root is equal to $0.15 \mathrm{rad}=8.5^{\circ}$. Librations with the same amplitude at the hydrogen bonded nitrogen position produce about six times lower effect $(0.3 \mathrm{MHz} / 1.8 \mathrm{MHz}=1 / 6 ; 20 \mathrm{kHz} / 6 \approx 3 \mathrm{kHz})$ than at the non-hydrogen bonded nitrogen position. This can be the reason why they are not observed at this nitrogen position.

Upon increasing the temperature from $157 \mathrm{~K}$ to $323 \mathrm{~K}$ the ${ }^{14} \mathrm{~N}$ quadrupole coupling constant at the hydrogen bonded nitrogen position increases for $90 \mathrm{kHz}\left(\Delta q_{z z}=-90 \mathrm{kHz}\right)$, whereas at the non-hydrogen bonded nitrogen position it decreases for $53 \mathrm{kHz}\left(\Delta q_{z z}=53 \mathrm{kHz}\right)$. According to expression (4) at this temperature change the population of the nitrogen $\pi$-electron orbital at the hydrogen bonded nitrogen positions decreases for 0.005 whereas at the non-hydrogen bonded nitrogen position it increases for 0.003 .

In cocrystals of 2,3,5,6-tetramethylpyrazine we also observed a correlation between the ${ }^{14} \mathrm{~N}$ quadrupole coupling constants at the hydrogen bonded and non-hydrogen bonded nitrogen positions. A lower quadrupole coupling constant at the hydrogen bonded nitrogen position results in a higher quadrupole coupling constant at the non-hydrogen bonded nitrogen position. Such a correlation is not observed in cocrystals of BPY.

\section{Conclusions}

Cocrystals BPY-BA $(1: 1)$, BPY-5CSA (1:1), BPY-OXA (1:1), BPY-MA (1:1), BPY-4HBA $(2: 1)$, and BPY-4HBA $(1: 2)$ were grown from the methanol solution of the cocrystal formers. The cocrystals BPY-OXA $(1: 1)$ were also recrystallized from the water solution.

Complete ${ }^{14} \mathrm{~N}$ NQR spectra of these cocrystals have been measured by ${ }^{1} \mathrm{H}^{14}{ }^{14}$ nuclear quadrupole double resonance. The principal values of the quadrupole coupling tensor are calculated from the ${ }^{14} \mathrm{~N}$ NQR frequencies. A large variation of ${ }^{14} \mathrm{~N}$ quadrupole coupling constant between $1.3 \mathrm{MHz}$ and $4.7 \mathrm{MHz}$ is observed. At the non-hydrogen bonded nitrogen position in a BPY molecule the ${ }^{14} \mathrm{~N}$ quadrupole coupling constant is around 4.6 MHz. An increasing strength of the $\mathrm{O}-\mathrm{H} \cdots \mathrm{N}$ hydrogen bond reduces the ${ }^{14} \mathrm{~N}$ quadrupole coupling constant. In the case of weak carboxylic acids BA and $4 \mathrm{HBA}$, the ${ }^{14} \mathrm{~N}$ quadrupole coupling constant is reduced to approximately $4 \mathrm{MHz}$. Polymorphism in BPY-4HBA $(1: 2)$ has not been observed. A somewhat stronger MA, which forms two equivalent hydrogen bonds with two BPY molecules, reduces the ${ }^{14} \mathrm{~N}$ quadrupole coupling constant to about 3.6 MHz. A still stronger 5CSA reduces ${ }^{14} \mathrm{~N}$ quadrupole coupling constant to about $2.5 \mathrm{MHz}$. A very low ${ }^{14} \mathrm{~N}$ quadrupole coupling constant, about $1.3 \mathrm{MHz}$, is observed in BPY-OXA (1:1). Such a low ${ }^{14} \mathrm{~N}$ quadrupole coupling constant is characteristic for proton transfer $\left(\mathrm{O}-\mathrm{H} \cdots \mathrm{N} \rightarrow \mathrm{O}^{-} \cdots \mathrm{H}-\mathrm{N}^{+}\right)$.

A correlation of the principal values of the ${ }^{14} \mathrm{~N}$ quadrupole coupling tensor is observed in the whole range from the noninteracting pyridine in the gas phase through the presently obtained data to the pyridinium ion. The correlation is analyzed in the model, where the deformation of the lone pair electron orbital and the change of the population of the $\pi$-electron orbital produce the variation of the ${ }^{14} \mathrm{~N}$ quadrupole coupling tensor in hydrogen bonded BPY.

The value of the ${ }^{14} \mathrm{~N}$ quadrupole coupling constant at the hydrogen bonded nitrogen position in BPY-5CSA $(1: 1)$ is in midway between the value observed at the non-hydrogen bonded nitrogen position $(\sim 4.6 \mathrm{MHz})$ and the value observed in the case of the pyridinium ion $(\sim 1 \mathrm{MHz})$. Such an intermediate value of the ${ }^{14} \mathrm{~N}$ quadrupole coupling constant is characteristic for a short, strong hydrogen bond. To check for the temperature variation of the proton position within the short, strong hydrogen bond we measured the ${ }^{14} \mathrm{~N}$ NQR frequencies at various temperatures between $157 \mathrm{~K}$ and $323 \mathrm{~K}$. The NQR data show that the proton migrates upon increasing the temperature in the direction from the hydrogen bond acceptor to the hydrogen bond donor. The proton displacement is in the temperature range between $157 \mathrm{~K}$ and $323 \mathrm{~K}$ equal to $0.03 \AA$.

From the temperature variation of the principal values of the ${ }^{14} \mathrm{~N}$ quadrupole coupling tensor at the two nitrogen positions in 
BPY-5CSA (1:1) we estimated the mean-square libration angle of the non-hydrogen bonded pyridine ring at room temperature and the change of the population of the $\pi$-electron orbital in the temperature interval between $157 \mathrm{~K}$ and $323 \mathrm{~K}$ at the two nitrogen positions in a BPY molecule.

\section{References}

1 G. J. Desiraju, J. Am. Chem. Soc., 2013, 135, 9952.

2 G. P. Stahly, Cryst. Growth Des., 2007, 7, 1007.

3 N. Schultheiss and A. Newman, Cryst. Growth Des., 2009, 9, 2950.

4 S. Horiuchi and Y. Tokura, Nat. Mater., 2008, 7, 357.

5 V. R. Pedireddi, S. Chatterjee, A. Ranganathan and C. N. R. Rao, Tetrahedron, 1998, 54, 9457.

6 J. A. Cowan, J. A. K. Howard, H. Puschmann and I. D. Williams, Acta Crystallogr., Sect. E: Struct. Rep. Online, 2007, 63, o1240.

7 J. A. Cowan, J. A. K. Howard, G. J. McIntyre, S. M.-F. Lo and I. D. Williams, Acta Crystallogr., Sect. B: Struct. Sci., 2003, 59, 794.

8 J. A. Cowan, J. A. K. Howard and M. A. Leech, Acta Crystallogr., Sect. C: Cryst. Struct. Commun., 2001, 57, 302.

9 M. B. Zaman, M. Tomura and Y. Yamashita, Chem. Commun., 1999, 999.

10 P. L. Coupar, G. Ferguson and C. Glidewell, Acta Crystallogr., Sect. C: Cryst. Struct. Commun., 1996, 52, 2524.

11 A. Mukherjee and G. R. Desiraju, Chem. Commun., 2011, 47, 4090.

12 J. Seliger, Acta Chim. Slov., 2011, 58, 471.

13 J. Seliger, V. Žagar and T. Asaji, Hyperfine Interact., 2013, 222, 1.

14 J. Seliger and V. Žagar, Solid State Nucl. Magn. Reson., 2012, 47-48, 47.
15 J. Seliger and V. Žagar, J. Phys. Chem. B, 2013, 117, 6946.

16 J. Seliger and V. Žagar, J. Phys. Chem. B, 2014, 118, 996.

17 See for example: J. Seliger, Nuclear quadrupole resonance: theory, in Encyclopedia of Spectroscopy and Spectrometry, ed. J. C. Lindon, G. E. Tranter and J. L. Holmes, Academic Press, San Diego, 2000, pp. 1672-1680.

18 P. Pyykko, Mol. Phys., 2008, 106, 1965.

19 J. Seliger and V. Žagar, J. Magn. Reson., 2008, 193, 54.

20 J. Seliger, V. Žagar and R. Blinc, J. Magn. Reson., Ser. A, 1994, 106, 214.

21 J. Seliger, V. Žagar and R. Blinc, Z. Naturforsch., A: Phys. Sci., 1994, 49, 31.

22 J. Seliger and V. Žagar, J. Phys. Chem. A, 2013, 117, 1651.

23 L. Giube and E. A. C. Lucken, Mol. Phys., 1966, 10, 273.

24 J. Seliger and V. Žagar, J. Phys. Chem. A, 2011, 115, 11652.

25 J. A. Cowan, J. A. K. Howard, G. J. McIntyre, S. M.-F. Lo and I. D. Williams, Acta Crystallogr., Sect. B: Struct. Sci., 2005, 61, 724 .

26 F. Fontaine-Vive, M. R. Johnson, G. J. Kearley, J. A. Cowan, J. A. K. Howard and S. F. Parker, J. Chem. Phys., 2006, 124, 234503.

27 G. V. Rubenacker and T. L. Brown, Inorg. Chem., 1980, 19, 392 .

28 I. J. F. Poplett, M. Sabir and J. A. S. Smith, J. Chem. Soc., Faraday Trans. 2, 1981, 77, 1651.

29 J. Seliger, V. Žagar, A. Zidanšek and R. Blinc, Chem. Phys., 2006, 331, 131.

30 J. Seliger, V. Žagar, T. Asaji and A. Konnai, Magn. Reson. Chem., 2008, 46, 756.

31 J. Seliger, V. Žagar, T. Asaji, K. Gotoh and H. Ishida, Phys. Chem. Chem. Phys., 2011, 13, 9165.

32 N. Heineking, H. Dreizler and R. Schwarz, Z. Naturforsch., 1986, 41a, 1210. 